\title{
The heliospheric modulation of cosmic ray protons during increased solar activity: effects of the position of the solar wind termination shock and of the heliopause
}

\author{
U. W. Langner ${ }^{1,2}$ and M. S. Potgieter ${ }^{2}$ \\ ${ }^{1}$ Institut für Theoretische Physik IV, Ruhr-Universität Bochum, 44780 Bochum, Germany \\ ${ }^{2}$ Unit for Space Physics and School of Physics, North-West University, 2520 Potchefstroom, South Africa
}

Received: 19 October 2004 - Revised: 28 February 2005 - Accepted: 3 March 2005 - Published: 3 June 2005

\begin{abstract}
The effects on the modulation of cosmic ray protons of different positions for the solar wind termination shock and for the heliopause are illustrated for moderate solar maximum conditions. This is done with a numerical model which includes diffusive termination shock acceleration, a heliosheath and drifts. The modulation is computed for the heliospheric equatorial plane and at $35^{\circ}$ heliolatitude and for both magnetic polarity cycles of the Sun. It was found that the differences between the modulation for the two solar polarity cycles are less significant at a heliolatitude of $35^{\circ}$ than in the equatorial plane. The modulation for the different heliopause positions are qualitatively similar, although there are clear quantitative differences which should be observable with the two Voyager spacecraft in the outer heliosphere. It is illustrated that the motion of the termination shock from $90 \mathrm{AU}$ to $100 \mathrm{AU}$, with the heliopause fixed at $120 \mathrm{AU}$, is not crucially important to global modulation. What is of primary importance is the location of the heliopause. It can also be concluded from the results that significant asymmetric modulation is to be expected between the up-wind and down-wind directions of the heliosphere but this may become measureable only when spacecraft move beyond the termination shock into the heliosheath.
\end{abstract}

Keywords. Interplanetary physics (Cosmic rays; Heliopause and solar wind termination) - Space plasma physics (Transport processes)

\section{Introduction}

The effects of changing the position of the solar wind termination shock (TS) and the position of the heliopause on the modulation of cosmic ray protons were illustrated for solar minimum conditions by Langner and Potgieter (2005). For this work the research is extended to include moderate solar maximum conditions since the Voyager spacecraft is

Correspondence to: $\mathrm{U}$. W. Langner

(ul@tp4.rub.de) presently in the vicinity of the TS during these conditions (e.g. Stone and Cummings, 2001; McDonald et al., 2003; Stone and Cummings, 2003; Krimigis et al., 2003). This study is done with a comprehensive numerical model which includes convection, diffusion, adiabatic energy changes, diffusive termination shock acceleration, a heliosheath and gradient and curvature drifts.

The TS is described as a collisionless shock, i.e. a discontinuous transition from supersonic to subsonic flow speed accompanied by discontinuous increases in the number density, temperature and pressure. For our purposes the heliopause which separates the solar and interstellar plasmas is considered as the outer modulation boundary. The region between the heliopause and the TS is called the heliosheath. For a review on the features of the outer heliosphere, see Fichtner (2001). There is a reasonable consensus that the TS should be in the vicinity of $(90 \pm 5)$ AU (e.g. Stone and Cummings, 2001) in the direction that the heliosphere is moving (heliospheric nose), although over a solar cycle the TS may move outwards and inwards (e.g. Whang et al., 2004). It is not expected that the TS position will be more than 90 100 AU away from the Sun towards the tail of the heliosphere so that the assumption of a spherical TS is still considered quite reasonable. The position of the heliopause, on the other hand, is less certain, probably at least 30-50 AU beyond the TS in the nose direction, but significantly further away in the tail direction. This is because the geometry of the heliosphere should be affected by the relative motion of the heliosphere through the local interstellar medium (e.g. Scherer and Fahr, 2003; Zank and Müller, 2003).

The purpose of this work is to extend the work of Langner and Potgieter (2005), and the work done by Ferreira et al. (2001) for cosmic ray electrons, in studying the effects on the modulation of cosmic ray protons of varying TS and heliopause positions, in particular during moderate solar maximum conditions, and for the up-wind and down-wind directions with respect to the interstellar wind. We restrict the study to the equatorial regions of the heliosphere because of the limitations of the two-dimensional model used 
for this study. First, we keep the TS position at $r_{s}=90 \mathrm{AU}$ but change the heliopause position from $r_{H P}=120 \mathrm{AU}$ to $r_{H P}=160$ AU. Second, we keep $r_{H P}=120$ AU but change $r_{s}=90 \mathrm{AU}$ to $r_{s}=100 \mathrm{AU}$. Third, we combine these positions to represent modulation in the up-wind and down-wind directions of the heliosphere during moderate solar maximum conditions. This study should help to interpret forthcoming observations of the Voyager spacecraft from a global modulation point of view.

\section{Modulation model}

The model is based on the numerical solution of the timedependent cosmic ray transport equation (Parker, 1965):

$$
\begin{aligned}
\frac{\partial f}{\partial t} & =-\left(\mathbf{V}+\left\langle\mathbf{v}_{D}\right\rangle\right) \cdot \nabla f+\nabla \cdot\left(\mathbf{K}_{S} \cdot \nabla f\right) \\
& +\frac{1}{3}(\nabla \cdot \mathbf{V}) \frac{\partial f}{\partial \ln p}+J_{\text {source }}
\end{aligned}
$$

where $f(\mathbf{r}, p, t)$ is the omnidirectional cosmic ray distribution function, $p$ is the particle momentum, $\mathbf{r}$ is position, and $t$ is time, with $\mathbf{V}$ the solar wind velocity. Terms on the righthand side represent convection, gradient and curvature drifts, diffusion, adiabatic energy changes and a source function, respectively. The tensor $\mathbf{K}_{S}$ consists of a parallel diffusion coefficient $\left(\kappa_{\|}\right)$, and perpendicular diffusion coefficients $\left(\kappa_{\perp}\right)$. The averaged guiding centre drift velocity for a near isotropic cosmic ray distribution is given by $\left\langle\mathbf{v}_{D}\right\rangle=\nabla \times\left(\kappa_{T} \mathbf{e}_{\mathbf{B}}\right)$, with $\mathbf{e}_{\mathbf{B}}=\mathbf{B} / B_{m}$, where $B_{m}$ is the magnitude of the modified background HMF as described below; here $\kappa_{T}$ (sometimes indicated as $\kappa_{A}$ ) is the diffusion coefficient specified by the off-diagonal elements of the generalized diffusion tensor $\mathbf{K}$, which describes gradient and curvature drifts in the largescale HMF. The function $J_{\text {source }}$ may represent any local source, such as Jovian electrons (e.g. Ferreira, 2002), pickup ions, anomalous cosmic rays, etc., which is not utilized for this work (i.e. $J_{\text {source }}=0$ ). The diffusion coefficients $\kappa_{\|}$, $\kappa_{\perp}$, and $\kappa_{T}$ are based on those given by Burger et al. (2000) for a steady-state model, except for changes caused by the introduction of the TS in this model (Langner et al., 2003). Perpendicular diffusion is assumed to enhance towards the poles. For a complete description of these diffusion coefficients and details of the model, see Langner et al. (2003) and Langner and Potgieter (2004a).

The transport equation is solved time-dependently in a spherical coordinate system as a combined diffusive shock acceleration and drift modulation model with two spatial dimensions, neglecting any azimuthal dependence and is symmetric with respect to the polar axis. A similar twodimensional numerical model was described originally by Jokipii et al. (1993); see also Potgieter and Ferreira (2002). The HMF was assumed to have a basic Parkerian geometry in the equatorial plane but was modified in the polar regions similar to the approach of Jokipii and Kóta (1989).

The position of the outer modulation boundary (heliopause) is assumed to be either at $r_{H P}=120 \mathrm{AU}$ or
$r_{H P}=160 \mathrm{AU}$, where the proton local interstellar spectrum (LIS) of Moskalenko et al. (2002) is specified. The position of the TS is assumed at $r_{s}=90 \mathrm{AU}$ and $r_{s}=100 \mathrm{AU}$, respectively, with a compression ratio $s=3.2$ and a shock precursor scale length of $L=1.2 \mathrm{AU}$ (see also Langner et al., 2003). This means that up to the shock, the solar wind speed $V$ decreases by $0.5 s$ starting at $L$, then abruptly as a step function to the downstream value, in total to $V / s$. Beyond the TS, $V$ decreases as $1 / r^{2}$ up to the outer boundary, which implies that no additional acceleration can occur beyond the shock and that adiabatic energy losses become insignificant, which may be an oversimplification.

Concerning the solar wind, it is assumed that $V$ has no clear latitudinal dependence typical for solar maximum conditions as was observed by Ulysses (McComas et al., 2000). It is therefore assumed to be on average $400 \mathrm{~km} \mathrm{~s}^{-1}$ in the whole heliosphere in contrast to solar minimum conditions when $V$ has a strong latitude dependence. A modified version of the current sheet model of Hattingh and Burger (1995) was used, which emulates the waviness of the current sheet in two spatial dimensions (see also Langner, 2004). The current sheet tilt angles $\alpha$ as calculated by Hoeksema (Wilcox Solar Observatory; courtesy of J. T. Hoeksema: http://wso.stanford.edu) were assumed to represent moderate solar maximum modulation conditions with $\alpha=75^{\circ}$ during $A>0$ (e.g. $\sim 1990-2001$ ) and $A<0$ (e.g. $\sim 1980$ 1990) solar magnetic polarity cycles. The case with $\alpha=10^{\circ}$ was studied by Langner and Potgieter (2005).

\section{Results and discussion}

The computed differential intensities for galactic protons are shown in Fig. 1 (left panels) as a function of kinetic energy for both magnetic polarity cycles and for moderate solar maximum conditions with $\alpha=75^{\circ}$. The spectra are shown in the equatorial plane $\left(\theta=90^{\circ}\right)$ at radial distances of $1 \mathrm{AU}$, $60 \mathrm{AU}$, and at the TS location, in this case with $r_{s}=90 \mathrm{AU}$. These spectra are repeated with $\theta=55^{\circ}$ (heliolatitude of $35^{\circ}$ which is approximately the Voyager spacecraft trajectory latitude). In the right-hand panels the corresponding differential intensities are shown as a function of radial distance for $0.016,0.2$ and $1.0 \mathrm{GeV}$, respectively. The LIS is specified at the heliopause, first assumed at $r_{H}=120 \mathrm{AU}$ (as a representation of the up-wind direction - the direction in which the heliosphere is moving relative to interstellar space) and then with $r_{H P}=160 \mathrm{AU}$ (as a representation of the down-wind direction - the tail region of the heliosphere). The purpose is to illustrate what effect this change in position has on the modulation of cosmic ray protons in the heliosheath and in the rest of the heliosphere. All the modulation parameters, including the diffusion coefficients, are kept the same for both situations.

The modulation for the different heliopause positions look qualitatively similar, but clearly differ quantitatively, especially when considered as a function of radial distance. The intensities with $r_{H P}=120 \mathrm{AU}$ in all four panels of Fig. 1 are 
higher than for the $160 \mathrm{AU}$ position as one would expect. The differences between the intensities at different AU are most prominent for energies $<\sim 1 \mathrm{GeV}$ and these differences increase with decreasing energy indicative of the wider heliosheath (larger modulation volume), especially during the $\mathrm{A}<0$ cycle. The total modulation between the heliopause and Earth is considerably more for the $\mathrm{A}<0$ cycle. These differences were more enhanced for solar minimum conditions, as reported by Langner and Potgieter (2005), than what we found here, as judged by the amount and at what energies for the $\mathrm{A}<0$ cycle the spectra at $90 \mathrm{AU}$ (and even at $60 \mathrm{AU}$ for solar minimum conditions) exceed the LIS value for energies $>\sim 2 \mathrm{GeV}$. In Fig. 1 the spectra at $90 \mathrm{AU}$ only are equal or somewhat larger than the LIS. As they reported, this "excess" effect is reduced when the heliopause is moved further out. As a function of radial distance these effects are quite evident for the chosen energies, e.g. the $0.20 \mathrm{GeV}$ intensities are lower at all radial distances. The "barrier" type effect, as manifested by the sharp drop in intensities over relatively small radial distances in the heliosheath, becomes more pronounced (covers a larger distance) when the heliopause is moved outward, especially during the $\mathrm{A}<0$ cycles when it happens over an extended energy range. The slowly decreasing effective radial diffusion coefficient with increasing radial distance beyond the TS contributes to this trend. The extent (width) of this modulation "barrier" is dependent on the values of the diffusion coefficients close to the outer boundary, and especially the direction of the drift velocities. For energies < $200 \mathrm{MeV}$ most of the modulation happens inside the heliosheath for both cycles, but especially because of the "barrier" covering relatively small distances near the heliopause during the $\mathrm{A}>0$ cycle. The conspicuous difference about what happens with the intensities at the TS between the two polarity cycles was discussed at length by Langner et al. (2003) and Langner and Potgieter (2004a, 2004b). Obviously, the very illustrative situation at $0.016 \mathrm{GeV}$ for galactic protons will be completely overshadowed by the presence of the anomalous protons which were not considered for this study; see Langner and Potgieter (2004a). Compared to the corresponding solar minimum situation reported by Langner and Potgieter (2005), the radial dependence of the modulation in the heliosheath is more gradual and the effects of the TS are less conspicuous, especially for the $\mathrm{A}<0$ cycle. Even the "barrier" effect is spread out over a larger distance for solar maximum conditions which qualitatively makes good sense. Inside the TS, the radial gradients are in all energy cases larger than for the solar minimum situation.

In the lower set of panels in Fig. 1 with $\theta=55^{\circ}$, the "barrier" effect is diminished because the total modulation in the heliosheath is less. As a function of radial distance the modulation in the heliosheath happens considerably more gradually over distance, especially the wider the heliosheath becomes. The radial dependence of the intensities in the heliosheath becomes rather similar for the two polarity cycles, and for the two heliopause situations, even at the TS, so that it is unlikely that in the future the two Voyager spacecraft will be able to distinguish between the two polarities using

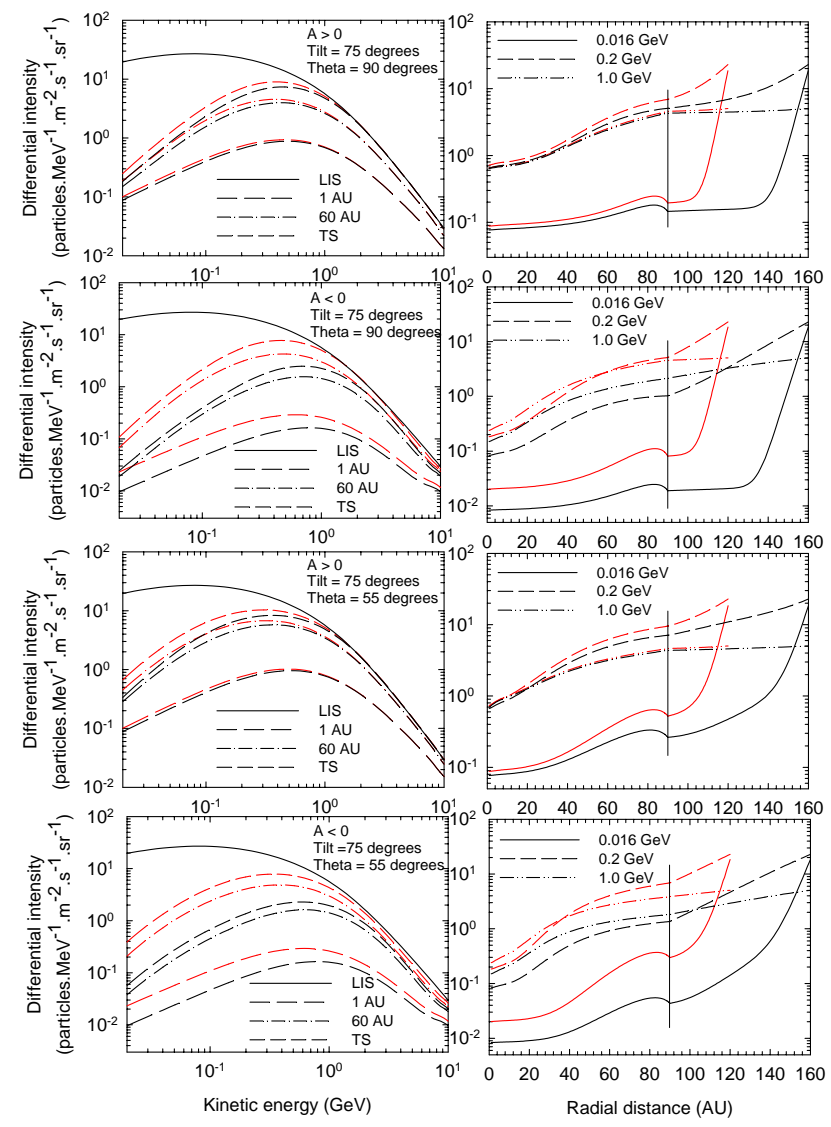

Fig. 1. Left panels: top two figures, computed differential intensities for galactic protons with $\alpha=75^{\circ}$ as a function of kinetic energy for both polarity cycles, at radial distances of $1 \mathrm{AU}, 60 \mathrm{AU}$ and the TS location (bottom to top) in the equatorial plane $\left(\theta=90^{\circ}\right)$. Bottom two figures: similar but at $\theta=55^{\circ}$. Right panels: corresponding differential intensities as a function of radial distance for 0.016, 0.2 and $1.0 \mathrm{GeV}$, respectively. The TS is at $90 \mathrm{AU}$, as indicated, with the heliopause at $120 \mathrm{AU}$ (red curves) and $160 \mathrm{AU}$ (black curves) respectively, where the LIS was specified.

only cosmic rays.

In Fig. 2 the heliopause position is maintained at $r_{H P}=120 \mathrm{AU}$ but the TS position is moved outwards from $90 \mathrm{AU}$ to $100 \mathrm{AU}$. Comparing the spectra for the two situations the intensities are lower at all energies of interest for $r_{s}=90 \mathrm{AU}$ than for $r_{s}=100 \mathrm{AU}$, even at Earth which was not as clear for solar minimum conditions. This means that moving the TS outwards, while keeping the heliopause positions and other parameters fixed, does not cause additional total modulation, although there are clear quantitative differences in the outer heliosphere. The effectiveness of the TS is also rather similar for the two scenarios. The exact position of the TS (given that it moves within $10 \mathrm{AU}$ or so) is somewhat more important for the moderate solar maximum scenario than for the solar minimum scenario, but this is still not crucially important to the total modulation.

Inspection of the radial dependence shown in the righthand panels of Fig. 2 emphasized what is shown for the 


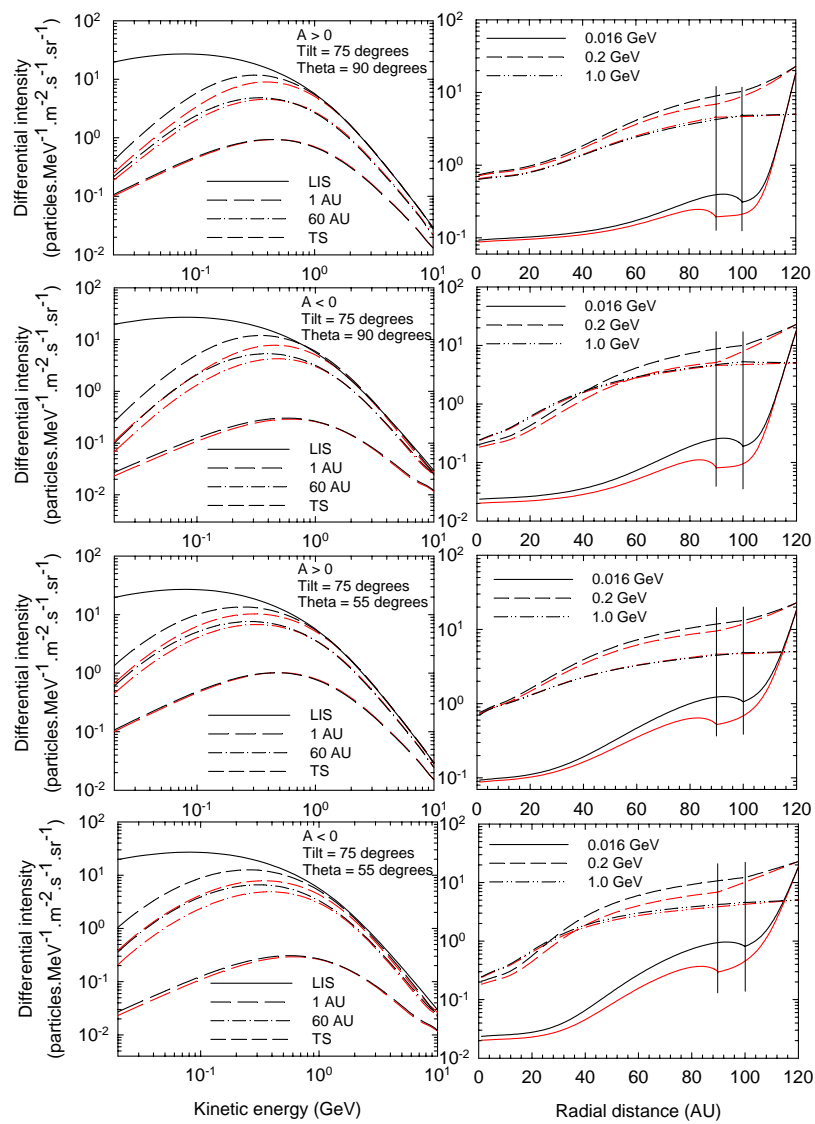

Fig. 2. Similar to Fig. 1 but with the TS positioned at $90 \mathrm{AU}$ (red curves) and $100 \mathrm{AU}$ (black curves), respectively, as indicated with the heliopause position specified at $120 \mathrm{AU}$.

spectra. The modulation trends inside the TS simply persist when it is shifted $10 \mathrm{AU}$ further out; the occurrence of heliosheath modulation characteristics are postponed until the TS is reached. From a diffusion point of view it must be noted that the diffusion coefficients keep on increasing with radial distance until the TS is reached so that the intensities will follow suit; the diffusion coefficients then drop at the TS by the value of the compression ratio, to decrease moderately with increasing distance. If the TS is positioned further out, the intensities must be higher as the $0.016 \mathrm{GeV}$ protons clearly illustrate. The "barrier" effect mentioned with Fig. 1 is not affected by moving the TS. As with Fig. 1, the differences between the two polarity cycles at $\theta=55^{\circ}$ are almost negligible and the heliosheath effects are significantly more moderate than in the equatorial regions.

Figure 3 illustrates two combined scenarios, the first with $r_{s}=90 \mathrm{AU}$ and $r_{H P}=120$, compared to $r_{s}=100 \mathrm{AU}$ with $r_{H P}=160 \mathrm{AU}$. The two cases are indicative of what can be expected, respectively, in the nose and the tail region of the heliosphere. Qualitatively, the results are similar to the previous two figures and are not discussed again in any detail. Note that if the TS and the heliopause "move" in the same direction, the effects on the modulation of protons are somewhat tempered. It can be concluded from these results that

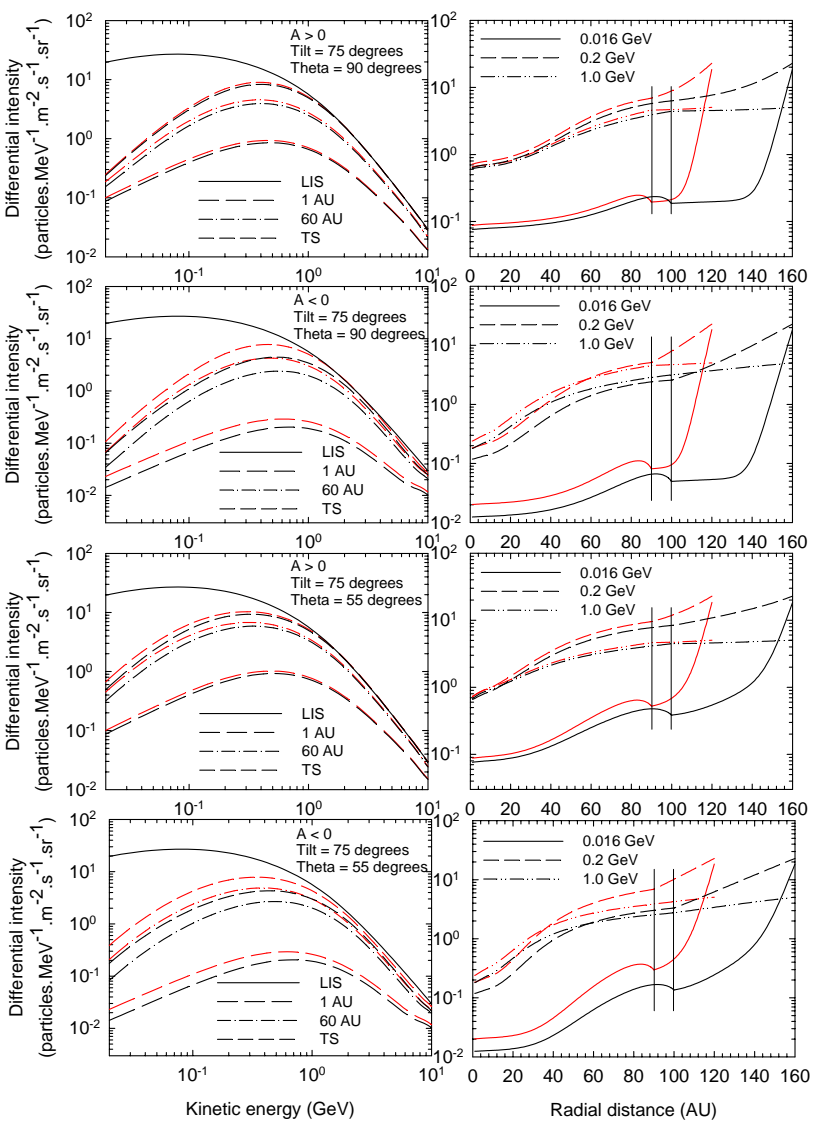

Fig. 3. Similar to Fig. 1 but with the TS positioned at 90 AU and with the heliopause at $120 \mathrm{AU}$ (red curves) compared to the TS at $100 \mathrm{AU}$ with the heliopause at $160 \mathrm{AU}$ (black curves).

significant asymmetric modulation can be expected between the up-wind and down-wind directions but these may become measureable only when spacecraft move somewhat beyond the TS into the heliosheath. These "asymmetry" effects will be further studied in the future using a true asymmetric modulation model based on a model originally developed by Haasbroek and Potgieter (1998).

As for solar minimum conditions it is quite evident from all three figures that the radial gradients of protons in the equatorial regions change significantly at the TS; for the A $>0$ cycle they become less beyond the shock at all energies of interest compared to inside the shock, while for the $\mathrm{A}<0$ cycle they increase in the heliosheath but only in the $100-500 \mathrm{MeV}$ range.

\section{Conclusions}

In a first of two papers, the effects of different positions of the TS and the heliopause on the heliospheric modulation of galactic protons for solar minimum conditions were illustrated by Langner and Potgieter (2005). A similar study was conducted by Potgieter and Ferreira (2002) for electrons. For the present study we elaborate on these effects 
but for moderate solar maximum conditions. This has become relevant because of the current positions of the two Voyager spacecraft, as well as the present activity phase of the Sun. A numerical model that includes particle drifts, the solar wind termination shock, a heliosheath beyond the shock, and a new approach to heliospheric diffusion coefficients has been used. This study was done for different scenarios of the position of the TS $\left(r_{s}=90\right.$ or $\left.100 \mathrm{AU}\right)$ and the heliopause $\left(r_{H P}=120\right.$ or $\left.160 \mathrm{AU}\right)$ to basically simulate the heliospheric equatorial regions in the nose (e.g. $r_{s}=90$; $\left.r_{H P}=120\right)$ and tail directions (e.g. $r_{s}=100 ; r_{H P}=160$ ). Previously, the model with $r_{s}=90 \mathrm{AU}$ and $r_{H P}=120 \mathrm{AU}$ had been applied successfully to the modulation of cosmic ray protons, anti-protons, electrons, positrons and $\mathrm{He}$, as discussed by Langner et al. (2003), Langner and Potgieter (2004a, 2004b), and Potgieter and Langner (2004).

It was found that the global modulation results for the different heliopause positions look qualitatively similar, but clearly differed quantitatively, especially when considered as a function of radial distance. The spectra in the equatorial plane for $r_{H P}=120 \mathrm{AU}$ were higher than for the $r_{H P}=160$ AU position with the differences between the differential intensities most prominent for energies $<\sim 1 \mathrm{GeV}$ and which increased with decreasing energy, indicative of the larger (wider) heliosheath. These differences were more enhanced for solar minimum conditions as reported by Langner and Potgieter (2005) than what we found here. The "barrier" effect close to the heliopause becomes more prominent when the heliopause was moved outwards, especially during the $\mathrm{A}<0$ cycles where it happens over an extended energy range. The extent of this modulation "barrier" is dependent on the value of the diffusion coefficients close to the outer boundary. At a heliolatitude of $35^{\circ}$ the radial dependence of the intensities in the heliosheath becomes very similar for the two polarity cycles, and for the two heliopause scenarios, so that it is unlikely that in the future the two Voyager spacecraft will be able to distinguish between the two polarities using only cosmic rays.

For the scenario where the TS positions were moved outwards from $r_{s}=90 \mathrm{AU}$ to $r_{s}=100 \mathrm{AU}$ with $r_{H P}=120 \mathrm{AU}$, the intensities for the first situation were lower at all energies of interest which seems counterintuitive. This means that moving the TS outwards while keeping the heliopause positions and other parameters fixed does not increase the total modulation between the heliopause and Earth. The exact position of the TS (given that it moves within $10 \mathrm{AU}$ or so) is therefore not crucially important to global modulation, although the effect can be larger for moderate solar maximum conditions than for solar minimum conditions. If the TS and the heliopause were moved in the same direction, the effects on the modulation became somewhat tempered.

The two cases, indicative of what can be expected, respectively in the nose and the tail region of the heliosphere, were also studied. It can be concluded from these results that significant asymmetric modulation can be expected between the heliospheric up-wind and down-wind directions but these differences may become measurable only when spacecraft move beyond the TS into the heliosheath. These "asymmetry" effects will be further studied in the future using a true asymmetric modulation model based on the model developed by Haasbroek and Potgieter (1998).

Acknowledgements. We thank H. Fichtner, B. Heber, S. Ferreira and K. Scherer for useful discussions. We thank the National Research Foundation (NRF) for partial financial support under the bilateral DFG/NRF agreement, GUN number 2049412.

Topical Editor R. Forsyth thanks K. Scherer and another referee for their help in evaluating this paper.

\section{References}

Burger, R. A., Potgieter, M. S., and Heber, B.: Rigidity dependence of cosmic-ray proton latitudinal gradients measured by the Ulysses spacecraft: Implications for the diffusion tensor, J. Geophys. Res., 105, 27 447-27 456, 2000.

Ferreira, S. E. S.: The heliospheric transport of galactic cosmic rays and jovian electrons, Ph.D. thesis, Potchefstroom University, South Africa, 2002.

Ferreira, S. E. S., Potgieter, M. S., and Lopate, C.: Consequences of different local interstellar spectra on $16 \mathrm{MeV}$ electron modulation, Proc. $27^{\text {th }}$ Inter. Cosmic Ray Conf. (Hamburg), 8, 36533656, 2001.

Fichtner, H.: Anomalous Cosmic Rays: Messengers from the outer heliosphere, Space Sci. Rev., 95, 639-754, 2001.

Haasbroek, L. J. and Potgieter, M. S.: A new model to study galactic cosmic ray modulation in an asymmetrically bounded heliosphere, J. Geophys. Res., 103, 2099-2104, 1998.

Hattingh, M., and Burger, R. A.: A new simulated wavy neutral sheet drift model, Adv. Space Res., 13(9), 213-216, 1995.

Jokipii, J. R. and Kóta, J.: The polar heliospheric magnetic field, Geophys. Res. Lett., 16, 1-4, 1989.

Jokipii, J. R., Kóta, J., and Merényi, E.: The gradient of galactic cosmic rays at the solar wind termination shock, Astrophys. J., 405, 782-786, 1993.

Krimigis, S. M., Decker, R. B., and Hill, et al.: Voyager 1 exited the solar wind at a distance of $\sim 85 \mathrm{AU}$ from the Sun, Nature, 426, 45-48, 2003.

Langner, U. W.: Effects of termination shock acceleration on cosmic rays in the heliosphere, Ph.D. thesis, Potchefstroom University, 2004.

Langner, U. W. and Potgieter, M. S.: Solar wind termination shock and heliosheath effects on the modulation of protons and anti-protons, J. Geophys. Res., 109(A01103), 1-12, doi:10.1029/2003JA010158, 2004a.

Langner, U. W. and Potgieter, M. S.: Effects of the solar wind termination shock and heliosheath on the heliospheric modulation of galactic and anomalous Helium, Ann. Geophys., 22, 3063-3072, 2004b,

SRef-ID: 1432-0576/ag/2004-22-3063.

Langner, U. W. and Potgieter, M. S.: Effects of the position of the solar wind termination shock and the heliopause on the heliospheric modulation of cosmic ray protons, Adv. Space Res., in press, 2005.

Langner, U. W., Potgieter, M. S., and Webber, W. R.: Modulation of cosmic ray protons in the heliosheath, J. Geophys. Res. 108(A10), 8039, LIS 14-1, doi:10.1029/2003JA009934, 2003. 
McComas, D. J.: Ulysses observations of the three-dimensional solar wind over the solar cycle and their implications for the outer heliosphere, EOS, 81, F983, 2000.

McDonald, F. B., Stone, E. C., and Cummings, et al.: Enhancement of energetic particles near the heliospheric termination shock, Nature, 426, 48-51, 2003.

Moskalenko, I. V., Strong, A. W., Ormes, J. F., and Potgieter, M. S.: Secondary antiprotons and propagation of cosmic rays in the galaxy and heliosphere, Astrophys. J., 565, 280-296, 2002.

Parker, E. N.: The passage of energetic charged particles through interplanetary space, Plan. Space Sci., 13, 9-49, 1965.

Potgieter, M. S. and Ferreira, S. E. S.: Effects of the solar wind termination shock on the modulation of Jovian and galactic electrons in the heliosphere, J. Geophys. Res., 107, SSH1, 1-9, 2002.

Potgieter, M. S. and Langner, U. W.: Heliospheric modulation of cosmic ray positrons and electrons: Effects of the heliosheath and the solar wind termination shock, Astrophys. J., 602, 9931001, 2004.
Scherer, K. and Fahr, H. J.: Solar cycle induced variations of the outer heliospheric structures, Geophys. Res. Lett., 30, 17: 1-4, doi:10.1029/2002GL016073, 2003.

Stone, E. C. and Cummings, A. C.: Estimate of the location of the solar wind termination shock, Proc. 27th Inter. Cosmic Ray Conf. (Hamburg), 10, 4263-4266, 2001.

Stone, E. C. and Cummings, A. C.: The approach of Voyager 1 to the termination shock. Proc. 28th Inter. Cosmic Ray Conf. (Tsukuba), 3889-3892, 2003.

Whang, Y. C., Burlaga, L. F., Wang, Y. M., and Sheeley, N. R.: The termination shock near $35^{\circ}$ latitude, Geophys. Res. Let., 31(L03805) doi:10.1029/2003GL018679, 2004.

Zank, G. P. and Müller, H. R.: The dynamical heliosphere, J. Geophys. Res., 108, SSH7, 1-15, doi:10.1029/2002JA009689, 2003. 